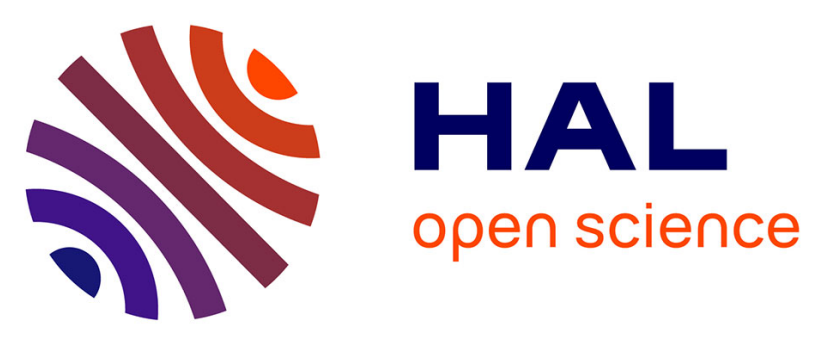

\title{
On Cross-risk Vulnerability
}

Yannick Malevergne, Rey Beatrice

\section{To cite this version:}

Yannick Malevergne, Rey Beatrice. On Cross-risk Vulnerability. Insurance: Mathematics and Economics, 2009, 45 (2), pp.224-229. 10.1016/j.insmatheco.2009.06.002 . halshs-00520050

\section{HAL Id: halshs-00520050 \\ https://shs.hal.science/halshs-00520050}

Submitted on 22 Sep 2010

HAL is a multi-disciplinary open access archive for the deposit and dissemination of scientific research documents, whether they are published or not. The documents may come from teaching and research institutions in France or abroad, or from public or private research centers.
L'archive ouverte pluridisciplinaire HAL, est destinée au dépôt et à la diffusion de documents scientifiques de niveau recherche, publiés ou non, émanant des établissements d'enseignement et de recherche français ou étrangers, des laboratoires publics ou privés. 


\title{
On Cross-Risk Vulnerability *
}

\author{
Y. Malevergne $e^{1,2,3}$ and B. Rey ${ }^{4}$ \\ ${ }^{1}$ University of Saint-Etienne - Institute of Business Administration \\ ${ }^{2}$ EM Lyon Business School - Cefra \\ ${ }^{3}$ ETH Zurich - Department Management, Technology and Economics \\ ${ }^{4}$ University of Lyon - University Lyon 1 - ISFA \\ ymalevergne@ethz.ch and rey-fournier@univ-lyon1.fr
}

\begin{abstract}
We introduce the notion of cross-risk vulnerability to generalize the concept of risk vulnerability introduced by Gollier and Pratt (1996). While risk vulnerability captures the idea that the presence of an unfair financial background risk should make risk-averse individuals behave in a more risk-averse way with respect to an independent financial risk, cross-risk vulnerability extends this idea to the impact of a non-financial background risk on the financial risk. It provides an answer to the question of the impact of a background risk on the optimal coinsurance rate and on the deductible level. We derive necessary and sufficient conditions for a bivariate utility function to exhibit cross-risk vulnerability both toward an actuarially neutral background risk and toward an unfair background risk. We also analyze the question of the sub-additivity of risk premia and show to what extent cross-risk vulnerability provides an answer.
\end{abstract}

Keywords: Risk aversion; Risk vulnerability; Multivariate risk; Background risk.

${ }^{*}$ The authors acknowledge helpful discussions and exchanges with L. Eeckhoudt. 


\section{Introduction}

Economic decision making under uncertainty often takes place in the context of multiple risks. Consequently, decisions about endogenous risks must often be taken while simultaneously facing one (or more) exogenous background risks. Hence, the introduction of multiple sources of uncertainty in the analysis of the demand for insurance represents significant progress (Doherty and Schlesinger, 1983a,b, 1986; Kischka, 1987; Eeckhoudt and Kimball, 1992, amongst others). Intuition suggests that, for a risk-averse individual, the presence of a background risk increases the risk aversion toward other independent risks. Therefore, concerning the demand for insurance, it should lead to an increase in the optimal coinsurance rate and to a decrease in the deductible level (Schlesinger, 1981).

However the literature shows that this natural relation between background risk and risk aversion is not always satisfied. Indeed, Gollier and Pratt (1996) have exhibited examples for which an undesirable lottery can be made desirable by the introduction of a mean-zero risk. For generalized expected utility preferences, Quiggin (2003) showed that aversion to one risk can be reduced by the presence of an independent background risk under the assumption of constant risk aversion in the sense of Safra and Segal (1998) and Quiggin and Chambers (1998). To avoid this counterfactual situation, Gollier and Pratt (1996) have provided conditions on preferences to guarantee that adding an unfair monetary background risk makes risk-averse individuals behave in a more risk-averse way. They name this restriction risk vulnerability to point out that the willingness to pay to bear risks is vulnerable to the introduction of another unfair risk. More recently, Franke et al. (2006) have analyzed the case of a multiplicative background risk and derived necessary and sufficient conditions on preferences to obtain a multiplicative-risk vulnerable utility function. In a different framework, Eichner and Wagener (2003) and Eichner (2008) have transfered the concept of risk-vulnerability into two-parameter mean-variance preferences.

All these results pertain to financial background risks only. The objective of this article is to extend the concept of risk vulnerability to the multivariate setting of the expected utility theory. It is all the more important that many economic problems often involve a background risk which is not of the same nature as the endogenous decision variables. We can, for example, refer to one-period models in which the background risk is a non-financial risk say, an environmental risk, or a health risk (Bleichrodt et al., 2003, for recent results). But the exogenous risk can also affect a non-consumable pension-asset, or the supply of labor, or an economic state variable, or the future consumption (Gollier, 2001; Eeckhoudt et al., 2005). We introduce the notion of cross-risk vulnerability to capture the idea that the presence of an unfair non-financial background risk should make risk-averse individuals behave in a more risk-averse 
way with respect to an independent financial risk. The knowledge of the set of cross-risk vulnerable utility functions is very useful insofar as, under cross-risk vulnerability, the presence of an unfair non pecuniary background risk increases the demand for insurance. In particular, background risk raises the optimal coinsurance rate and reduces the optimal level of deductible (for any given coinsurance rate). The presence of an unfair non pecuniary background risk also reduces the demand for risky assets.

The paper also relates the concept of cross-risk vulnerability to the properties underpinning the assumption of the preference for the disaggregation of harms. In the case of an additive background risk, Eeckhoudt and Schlesinger (2006) have shown that the preference for the disaggregation of harms is a necessary condition for a utility function to be risk vulnerable. In other words, the condition of risk vulnerability implies the necessity of the alternation of the signs of the first four derivatives of the utility function and therefore of the preference for the disaggregation of harms. The prudence (positive sign of the third derivative of the utility function) is thus a necessary condition to obtain risk vulnerability. We note that the concept of prudence is particularly important in many analyses relative to the insurance domain. In particular prudence plays an important role for the analysis of self-protection behaviors and for the evaluation of changes in survival probabilities (Chiu, 2005; Bleichrodt and Eeckhoudt, 2006). In this paper, we show that prudence (more precisely cross-prudence) still plays a decisive role. Besides, we show that the relation of inclusion between the set of risk vulnerable utility functions and the set of utility functions that satisfy the preference for the disaggregation of harms does not hold anymore for cross-risk vulnerability. The class of cross-risk vulnerable bivariate utility functions is thus less restrictive vis-a-vis the direction of the preferences for the aggregation or the disaggregation of harms.

To emphasize the importance of the notion of cross-risk vulnerability, we provide an application of our results for an individual who faces two independent risks. We wonder whether the risk premium is sub-additive. More precisely, we compare the total risk premium, i.e., the amount of money the agent agrees to pay to get rid of both risks, with the sum of the partial risk premia, i.e., the sum of the amounts of money the agent agrees to pay to get rid of each individual risk. This question has been previously investigated in a bivariate setting by Courbage (2001) in the limit of small risks, and by Rey (2003) for Bernoullian variables. We show here, without any restriction on the distributional properties of both risks, how cross-risk vulnerability is related to the relative ranking of the total risk premium and the sum of the partial risk premia.

Our paper is organized as follows. In section 2, we define the concept of cross-risk vulnerability. Then, in section 3, we provide a necessary and sufficient condition for a bivariate utility function to enjoy this property. Sections 4 and 5 offers more tractable conditions to obtain the cross-risk vulnerability 
property, and discusses the consistency of the assumptions often made on cross-derivatives of the utility function. We also show the link between the concept of cross-risk vulnerability and the properties behind the assumption of the preference for the disaggregation of harms. Section 6 presents our application of the cross-risk vulnerability to the relative ranking of the total risk premium and the sum of the partial risk premia. Some final comments are provided in the last section.

\section{Definition of cross-risk vulnerability}

We consider an individual with a two-argument von Neumann-Morgenstern utility function $U$ (assumed increasing and concave with respect to each argument, and $n$-times continuously differentiable). We will assume, for illustrative purposes and for ease of the exposition, that the two arguments refer to the agent's wealth and to her health respectively. Thus, we will denote by $\mathrm{E}\left[U\left(x_{0}+\tilde{z}, y_{0}+\tilde{\varepsilon}\right)\right]$ the individual's expected utility where $\left(x_{0}, y_{0}\right)$ represents her initial endowment, $\tilde{z}$ is a random wealth payoff and $\tilde{\varepsilon}$ a risk on the agent's health. We assume that these two risks are independent.

We address the question of the effect of the unfair background risk $\tilde{\varepsilon}$ - namely a risk with a nonpositive expectation, $\mathrm{E}[\tilde{\varepsilon}] \leq 0-$ on the willingness to pay to eliminate the independent financial risk $\tilde{z}$. We first introduce the partial bivariate risk premium for risk $\tilde{z}$ denoted $\pi^{1}$ (Chalfant and Finkelshtain, 1993). This risk premium is the maximum amount the individual agrees to give up in order to substitute the risk $\tilde{z}$ for its expected value in the presence of the risk $\tilde{\varepsilon}$. The risk premium is obtained as the solution to the following equation

$$
\mathrm{E}\left[U\left(x_{0}+\tilde{z}, y_{0}+\tilde{\varepsilon}\right)\right]=\mathrm{E}\left[U\left(x_{0}+\mathrm{E}(\tilde{z})-\pi^{1}, y_{0}+\tilde{\varepsilon}\right)\right]
$$

Obviously, $\pi^{1}$ explicitly depends upon $x_{0}, y_{0}, \tilde{z}$ and $\tilde{\varepsilon}$. For reasons of notational convenience, we write the dependence explicitly only when necessary. We want to compare $\pi^{1}$ with the risk premium for risk $\tilde{z}$ in the absence of the background risk $\tilde{\varepsilon}$ which is denoted by $\pi^{0}$ and is solution to the following equation

$$
\mathrm{E}\left[U\left(x_{0}+\tilde{z}, y_{0}\right)\right]=U\left(x_{0}+\mathrm{E}(\tilde{z})-\pi^{0}, y_{0}\right)
$$

We generalize the notion of derived utility function introduced by Kihlstrom et al. (1981) and Nachman 
(1982) to define the cross-derived utility function ${ }^{1} V$ as

$$
V(x, y)=\mathrm{E}[U(x, y+\tilde{\varepsilon})], \quad \forall x, y,
$$

so that equation (1) becomes

$$
\mathrm{E}\left[V\left(x_{0}+\tilde{z}, y_{0}\right)\right]=V\left(x_{0}+\mathrm{E}(\tilde{z})-\pi^{1}, y_{0}\right)
$$

Therefore, applying a well-known results due to Pratt (1964), it is equivalent to compare $\pi^{1}$ with $\pi^{0}$ and to compare the absolute risk aversion toward the financial risk for the derived utility function $V$ with the absolute risk aversion for the original utility function $U$.

Let us denote by $A(x, y):=-\frac{U_{11}(x, y)}{U_{1}(x, y)}$ the absolute risk aversion toward the financial risk (the absolute risk aversion on $x$ ) for the original utility function $U$. In order to determine whether $\pi^{1}$ is larger than $\pi^{0}$ we have to wonder whether the absolute risk aversion for the cross-derived utility function $V$

$$
A^{V}(x, y):=-\frac{\mathrm{E}\left[U_{11}(x, y+\tilde{\varepsilon})\right]}{\mathrm{E}\left[U_{1}(x, y+\tilde{\varepsilon})\right]}
$$

is larger than $A(x, y)$, for all $(x, y)$ and for all unfair risk $\tilde{\varepsilon}$. As a consequence, generalizing the notion of risk vulnerability introduced by Gollier and Pratt (1996) for additive risks and by Franke et al. (2006) for multiplicative risks, we propose the following definition:

Definition 1. The utility function $U$ is cross-risk vulnerable if (and only if) any unfair non-pecuniary background risk makes the agent behave in a more risk-averse way:

$$
\mathrm{E}[\tilde{\varepsilon}] \leq 0 \Longrightarrow A^{V}(x, y) \geq A(x, y) \quad \forall(x, y)
$$

Definition 1 suggests that any cross-risk vulnerable utility function is cross-DARA - i.e the risk aversion in wealth is decreasing in health, which reads $A_{2}(x, y) \leq 0$. Indeed, considering the degenerated background risk $\tilde{\varepsilon}=\varepsilon \in \mathbb{R}_{-}$, definition 1 yields

$$
A^{V}(x, y)=A(x, y+\varepsilon) \geq A(x, y) \quad \forall(x, y) \text { and } \forall \varepsilon \leq 0
$$

Therefore, the cross-DARA property is necessary for a utility function to be cross-risk vulnerable. Given

\footnotetext{
${ }^{1}$ The cross-derived utility function exhibits the same features as the one-dimensional derived utility function, in particular in terms of monotony and concavity with respect to each argument.
} 
the cross-DARA property, we can show that definition 1 is equivalent to the cross-risk vulnerability toward an actuarially neutral background risk, i.e, such that $\mathrm{E}[\tilde{\varepsilon}]=0$. More formally, definition 1 can be equivalently written as follows:

Definition 2. The cross-DARA utility function $U$ - i.e., such that $A_{2} \leq 0-$ is cross-risk vulnerable if (and only if) any actuarially neutral background risk makes the agent behave in a more risk-averse way:

$$
\mathrm{E}[\tilde{\varepsilon}]=0 \Longrightarrow A^{V}(x, y) \geq A(x, y) \quad \forall(x, y)
$$

The necessity is obvious from definition 1, while the sufficiency follows from the fact that any unfair background risk $\tilde{\varepsilon}$ can be expressed as $\bar{\varepsilon}+\tilde{\varepsilon}^{\prime}$ with $\mathrm{E}\left[\tilde{\varepsilon}^{\prime}\right]=0$ and $\mathrm{E}[\tilde{\varepsilon}]=\bar{\varepsilon}$, from which we obtain $A^{V}(x, y)=A^{V^{\prime}}(x, y+\bar{\varepsilon})$, where $A^{V^{\prime}}$ denotes the absolute risk aversion of the derived utility function $V^{\prime}$ obtained by taking the expectation in (3) with respect to $\tilde{\varepsilon}^{\prime}$ instead of $\tilde{\varepsilon}$. Thus, given an actuarially neutral background risk $\tilde{\varepsilon}^{\prime}$, definition 1 asserts that $U$ is cross-risk vulnerable if $A^{V^{\prime}}(x, y) \geq A(x, y)$,

$\forall x, y$. So, given any $\bar{\varepsilon} \leq 0, A^{V^{\prime}}(x, y+\bar{\varepsilon}) \geq A(x, y), \forall x, y$, since $A$ is decreasing in its second argument. Thus, for any unfair background risk $\tilde{\varepsilon}=\bar{\varepsilon}+\tilde{\varepsilon}^{\prime}$, we have $A^{V}(x, y) \geq A(x, y), \forall x, y$, which shows the sufficiency of the cross-risk vulnerability toward an actuarially neutral background risk.

In the remainder of this article, we will restrict our attention to cross-DARA utility functions. From a theoretical point of view, this assumption is not actually limiting. Indeed, as detailed latter on, the cross-DARA assumption just implies that the agent in cross-prudent in the second attribute, $U_{112} \geq 0$, provided the common assumption of correlation aversion, $U_{12} \leq 0$, holds (Epstein and Tanny, 1980; Richard, 1975). Besides, it does not place any restriction on the preference for the disaggregation of harms (Eeckhoudt et al., 2007). In addition, in the practical context of health economics for instance, even thought no empirical evidence exists on the relationship between patient's perception of health risks and their wealth, healthier people seem to be less adverse toward financial risks (see Bleichrodt $e t$ al. (2003) for a similar assumption).

\section{Characterization of cross-risk vulnerable utility functions}

The proposition bellow states the technical condition which fully characterizes the set of utility functions that satisfy the condition of cross-risk vulnerability: 
Proposition 1. A cross-DARA utility function $U$ is cross-risk vulnerable if and only if:

$$
\xi\left(x, y, y^{\prime}\right)=U_{1}\left(x, y^{\prime}\right)\left[A\left(x, y^{\prime}\right)-A(x, y)\right]-U_{1}(x, y) A_{2}(x, y)\left(y^{\prime}-y\right) \geq 0, \quad \forall x, y, y^{\prime} .
$$

The proof is given in appendix A. It reveals that, if the relation $\mathrm{E}[\tilde{\varepsilon}] \leq 0 \Longrightarrow A^{V}(x, y) \geq$ $A(x, y), \forall(x, y)$ is equivalent to $\xi\left(x, y, y^{\prime}\right) \geq 0$, the relation $\mathrm{E}[\tilde{\varepsilon}] \leq 0 \Longrightarrow A^{V}(x, y) \leq A(x, y), \forall(x, y)$ is equivalent to $\xi\left(x, y, y^{\prime}\right) \leq 0$. This remark stresses that when the constraint (9) does not hold, the introduction of an unfair background risk can actually lower the risk aversion.

Let us stress that proposition 1 allows one to retrieve the results given by Gollier and Pratt (1996) for additive risk vulnerability and by Franke et al. (2006) for multiplicative risk vulnerability. Indeed, considering the utility $U(x, y)=u(x+y)$, which accounts for additive risks, proposition 1 shows that $u$ is risk vulnerable toward an additive background risk if (and only if)

$$
\xi\left(x, y, y^{\prime}\right)=u^{\prime}\left(x+y^{\prime}\right)\left[r\left(x+y^{\prime}\right)-r(x+y)\right]-\left(y^{\prime}-y\right) u^{\prime}(x+y) r^{\prime}(x+y) \geq 0,
$$

where $r=-u^{\prime \prime} / u^{\prime}$ denotes the absolute risk aversion of $u$. Thus, if we replace $x+y$ by $w$ and $x+y^{\prime}$ by $x$, the previous relation yields

Corollary 1 (Gollier and Pratt (1996), Proposition 2). Utility function $u$ is additively risk vulnerable if and only if $u^{\prime}(x)[r(x)-r(w)]-(x-w) u^{\prime}(w) r^{\prime}(w) \geq 0$, for all $x, w$.

Similarly, we can consider the utility function $U(x, y)=u(x \cdot y)$ with $x, y \geq 0$ to take into account to case of multiplicative background risks. In such a situation, proposition 1 shows that $u$ is risk vulnerable toward a multiplicative background risk if and only if

$$
\xi\left(x, y, y^{\prime}\right)=y^{\prime} u^{\prime}\left(x y^{\prime}\right)\left[R\left(x y^{\prime}\right)-R(x y)\right]-x y u^{\prime}(x y) R^{\prime}(x y)\left(y^{\prime}-y\right) \geq 0
$$

where $R(x)=x \cdot r(x)$ is the relative risk aversion of $u$. The change of variables $x \rightarrow \frac{z}{y}$ and $y^{\prime}, \rightarrow y t$ leads to

$$
y \cdot\left(t u^{\prime}(z t)[R(z t)-R(z)]-z u^{\prime}(z) R^{\prime}(z)(t-1)\right) \geq 0,
$$

and since $y$ is positive, we finally get:

Corollary 2 (Franke et al. (2006), Main Theorem). Utility function u is multiplicatively risk vulnerable if and only if $u^{\prime}(z t)[R(z t)-R(z)]-z u^{\prime}(z) R^{\prime}(z)(t-1) \geq 0$, for all $t, z \geq 0$. 
As condition (9) is a priori neither very easy to interpret nor very easy to handle, we will provide in the following sections first necessary conditions and then sufficient conditions whose economic implications are more straightforward.

\section{Necessary conditions for cross-risk vulnerability}

Let us remark that $\xi(x, y, y)=0$ and $\left.\frac{\partial \xi}{\partial_{y^{\prime}}}\right|_{y^{\prime}=y}=0$ for all $(x, y)$, so that a necessary condition for (9) to hold is

$$
\left.\frac{\partial^{2} \xi}{\partial y^{\prime 2}}\right|_{y^{\prime}=y}=U_{1}(x, y) A_{22}(x, y)+2 U_{12}(x, y) A_{2}(x, y) \geq 0 .
$$

After expansion, this relation turn out to be equivalent to

$$
\frac{U_{1122}}{U_{11}} \geq \frac{U_{122}}{U_{1}}
$$

This necessary global condition is in fact a local necessary and sufficient condition ${ }^{2}$ whose intuitive means is obvious in the case of an individual who is both risk-averse toward financial risks $\left(U_{11}<0\right)$ and cross-prudent in wealth ${ }^{3}\left(U_{122} \geq 0\right)$. In such a case, relation (14) becomes

$$
-\frac{U_{1122}}{U_{122}} \geq-\frac{U_{11}}{U_{1}}
$$

Thus, an individual who is risk-averse and cross-prudent in wealth, is cross-risk vulnerable toward an actuarially neutral background risk (in the limit of small risk), if and only if her absolute risk aversion toward a financial risk is smaller than the absolute risk aversion of the function $U_{22}$, seen as a utility function per se. Alternatively, for an imprudent individual $\left(U_{122} \leq 0\right)$, cross-risk vulnerability toward

\footnotetext{
${ }^{2}$ Restricting our attention to cross-DARA utility functions and considering the impact of an actuarially neutral background risk $\tilde{\varepsilon}$ on the risk aversion with respect to the agent's wealth in the limit of small (non-financial) risks, the relative change in risk aversion reads

$$
\left.\frac{\Delta A}{A}\right|_{(x, y)}:=\frac{A^{V}(x, y)-A(x, y)}{A(x, y)} \simeq\left[\frac{U_{1122}}{U_{11}}-\frac{U_{122}}{U_{1}}\right] \cdot \frac{\sigma_{\tilde{\varepsilon}}^{2}}{2},
$$
}

where $\sigma_{\tilde{\varepsilon}}^{2}$ is the variance of $\tilde{\varepsilon}$, since up to the second order,

$$
A^{V}(x, y) \simeq A(x, y) \cdot\left(1+\left[\frac{U_{1122}}{U_{11}}-\frac{U_{122}}{U_{1}}\right] \cdot \frac{\sigma_{\tilde{\varepsilon}}^{2}}{2}\right) .
$$

Thus, the utility function $U$ is cross-risk vulnerable toward an actuarially neutral background risk (in the limit of small risks), if and only if

$$
\frac{U_{1122}}{U_{11}} \geq \frac{U_{122}}{U_{1}}
$$

${ }^{3}$ Following the denomination introduced by Bleichrodt et al. (2003) and Eeckhoudt et al. (2007), we say that an individual is cross-prudent in wealth if $U_{122} \geq 0$. 
an actuarially neutral background risk is equivalent to the fact that the agent's absolute risk aversion is larger than the absolute risk aversion of the function $U_{22}$, seen as a utility function per se.

We would like to stress that on the contrary to the case of an additive risk considered by Gollier and Pratt (1996), the class of cross-risk vulnerable utility functions is not merely a subset of the class of utility functions that comply with the preference for the disaggregation of harms (Eeckhoudt et al., 2007). Recall that for the one-argument utility function $u(x)$, the preference for the disaggregation of harms implies the alternation of the signs of the four first derivative of the utility function (Eeckhoudt and Schlesinger, 2006). Then, for an additive background risk, the risk-vulnerability vis-a-vis small risks reads

$$
-\frac{u^{\prime \prime \prime}}{u^{\prime \prime}} \geq-\frac{u^{\prime \prime}}{u^{\prime}} \geq 0 \quad \text { and } \quad-\frac{u^{\prime \prime \prime \prime}}{u^{\prime \prime \prime}} \geq-\frac{u^{\prime \prime}}{u^{\prime}} \geq 0
$$

which implies the alternation of the signs the four first derivative of $u(x)$.

In the case of a two-argument utility function $U(x, y)$, the preference for the disaggregation of harms requires that $U_{12} \leq 0, U_{112} \geq 0, U_{122} \geq 0$ and $U_{1122} \leq 0$ (Eeckhoudt et al., 2007). But, the crossDARA condition together with (14) are not enough to satisfy these requirements. Unless $U_{12} \leq 0$, i.e $U$ is correlation-averse ${ }^{4}$, the cross-DARA condition is not enough to assert that $U_{112}$ must be positive. Moreover, condition (14) does not imply that $U_{1122}$ must be negative unless we assume $U_{122} \leq 0$. As a consequence, given $U(x, y)$ is cross-risk vulnerable, the alternation of the signs of the first four order cross-derivatives of the utility function requires the minimal assumption that $U$ is correlation-averse and that $U_{122}$ and $U_{112}$ have the same sign. Consequently, cross-risk vulnerability is less restrictive vis-a-vis the direction of the preferences for the aggregation of the disaggregation of harms.

\section{Sufficient conditions for cross-risk vulnerability}

According to equation (14), a local sufficient condition for cross-risk vulnerability toward actuarially neutral small background risks is $U_{122} \leq 0$ and $U_{1122} \leq 0$, provided that the usual conditions $U_{1}>0$ and $U_{11}<0$ hold. It means that, even if imprudent in wealth $\left(U_{112} \leq 0\right)$, an individual can still be cross-risk vulnerable as long as she remains cross-temperate ${ }^{5}$. As stated by the proposition below, this locally sufficient condition is also globally sufficient:

\footnotetext{
${ }^{4}$ See Epstein and Tanny (1980) and Richard (1975). Remark that the sign of $U_{12}$ can be easily interpreted in relation with the sensitivity of the marginal utility of wealth with respect to health which depends on the severity of the illness (Evans and Viscusi, 1991; Sloan et al., 1998, e.g.).

${ }^{5}$ Again, following the denomination introduced by Bleichrodt et al. (2003) and Eeckhoudt et al. (2007), we say that an individual is cross-temperate if $U_{1122} \leq 0$.
} 
Proposition 2. The cross-DARA utility function $U(x, y)$ is cross-risk vulnerable if $U_{22}(x, y)$ is decreasing and concave in $x$, i.e. if $U_{122} \leq 0$ and $U_{1122} \leq 0$.

Proof. By Jensen's inequality

$$
-\frac{\mathrm{E}\left[U_{11}(x, y+\tilde{\varepsilon})\right]}{\mathrm{E}\left[U_{1}(x, y+\tilde{\varepsilon})\right]} \geq-\frac{U_{11}(x, y+\mathrm{E}[\tilde{\varepsilon}])}{U_{1}(x, y+\mathrm{E}[\tilde{\varepsilon}])}
$$

if $U_{1}$ and $U_{11}$ are concave functions of $y$, namely if $U_{122} \leq 0$ and $U_{1122} \leq 0$. Now, since $U$ is crossDARA, $A(x, y)$ is decreasing in $y$, so that

$$
-\frac{U_{11}(x, y+\mathrm{E}[\tilde{\varepsilon}])}{U_{1}(x, y+\mathrm{E}[\tilde{\varepsilon}])} \geq-\frac{U_{11}(x, y)}{U_{1}(x, y)}, \quad \forall \mathrm{E}[\tilde{\varepsilon}] \leq 0 .
$$

We observe that the condition in proposition 2 cannot be met if the agent is correlation averse $\left(U_{12} \leq 0\right)$ and if $U$ is only defined for $y \geq 0$. Indeed, as shown by Menegatti (2001), given a positive function $f(t)$ defined for $t \geq 0$ with $f^{\prime}(t)<0$, if $f^{\prime \prime}$ keeps a constant sign, it must be positive. So, applying this result to the positive function $f_{x}(t) \mapsto U_{1}(x, t)$, if $U_{12}(x, t)=f_{x}^{\prime}(t)<0$ we could not have $U_{122} \leq 0$ since it would means that $f_{x}^{\prime \prime}(t) \leq 0$ which contradicts Menegatti's result.

Another sufficient condition of the cross-risk vulnerability is the following.

Proposition 3. The cross-DARA utility function $U(x, y)$ is cross-risk vulnerable if $U_{12} \leq 0$ and $A_{22} \geq 0$, for all $x$ and $y$.

Proof. Since $U$ is cross-DARA, we have $A_{2} \leq 0$ so that, together with the correlation aversion, i.e. $U_{12} \leq 0$ we get $U_{12} \cdot A_{2} \geq 0$, from which we conclude that the random variables $U_{1}(x, y+\tilde{\varepsilon})$ and $A(x, y+\tilde{\varepsilon})$ are co-monotonic and therefore are positively correlated (Hotelling, 1936):

$$
\mathrm{E}\left[A(x, y+\tilde{\varepsilon}) \cdot U_{1}(x, y+\tilde{\varepsilon})\right] \geq \mathrm{E}[A(x, y+\tilde{\varepsilon})] \cdot \mathrm{E}\left[U_{1}(x, y+\tilde{\varepsilon})\right]
$$

Then, the convexity of $A$ in its second argument and Jensen's inequality allows us to conclude that

$$
\begin{aligned}
\mathrm{E}\left[A(x, y+\tilde{\varepsilon}) \cdot U_{1}(x, y+\tilde{\varepsilon})\right] & \geq A(x, y+\mathrm{E}[\tilde{\varepsilon}]) \cdot \mathrm{E}\left[U_{1}(x, y+\tilde{\varepsilon})\right] \\
& \geq A(x, y) \cdot \mathrm{E}\left[U_{1}(x, y+\tilde{\varepsilon})\right]
\end{aligned}
$$

since $\mathrm{E}[\tilde{\varepsilon}] \leq 0$. Now, since $A(x, y) \cdot U_{1}(x, y)=-U_{11}(x, y)$, we obtain the expected result.

Proposition 3 means that the correlation aversion together with the convexity of the absolute risk 
aversion (with respect to its second argument) are sufficient for the cross-risk vulnerability to hold. The later requirement is rather natural in so far as it means that the reduction of the risk premium $\pi^{1}$ due to an increase in health is a decreasing function of health.

We have to stress that the sufficient conditions of proposition 3 are very close to the necessity. Indeed, recall that from the necessary condition (13), we have

$$
A_{22}+2 \frac{U_{12} \cdot A_{2}}{U_{1}} \geq 0
$$

for any cross-risk vulnerable utility function. Thus, for a utility function such that either $U_{12}$ or $A_{2}$ is vanishingly small, we need $A_{22} \geq 0$. Conversely, if $A_{22}$ is vanishingly small, the product $U_{12} \cdot A_{2}$ must non-negative and since $U$ must be cross-DARA $\left(A_{2} \leq 0\right)$, we need $U_{12} \leq 0$.

As a consequence of proposition 3, we can provide an example of family of utility functions that enjoy the property of cross-risk vulnerability.

Corollary 3. Any function

$$
U(x, y)=-\exp [f(x) \cdot g(x)]
$$

where $f$ and $g$ are any positive, decreasing and convex functions, defines a cross-risk vulnerable utility function.

The proof is straightforward and left to the reader. As a first example, one can consider $f(x)=$ $\left(\frac{x}{x_{0}}\right)^{-\alpha}$ and $g(y)=\left(\frac{y}{y_{0}}\right)^{-\beta}$ with $\alpha$ and $\beta$ positive. Corollary 3 shows that the utility function

$$
U(x, y)=-\exp \left[\frac{x_{0}^{\alpha} \cdot y_{0}^{\beta}}{x^{\alpha} \cdot y^{\beta}}\right], \quad \forall(x, y) \in \mathbb{R}_{+} \times \mathbb{R}_{+}
$$

is cross-risk vulnerable. Another example can be provided by the case $f(x)=\exp \left[-\frac{x}{x_{0}}\right]$ and $g(y)=$ $\exp \left[-\frac{y}{y_{0}}\right]$, with $x_{0}$ and $y_{0}$ positive, which yields

$$
U(x, y)=-\exp \left[\exp \left[-\left(\frac{x}{x_{0}}+\frac{y}{y_{0}}\right)\right]\right], \quad \forall(x, y) \in \mathbb{R} \times \mathbb{R} .
$$

\section{Application}

We consider an individual who faces two independent risks. The first one is the financial risk $\tilde{z}$, while the second one is the non-financial risk $\tilde{\varepsilon}$ (a health risk, for example). The total risk premium $\pi$, solution 
to

$$
\mathrm{E}\left[U\left(x_{0}+\tilde{z}, y_{0}+\tilde{\varepsilon}\right)\right]=U\left(x_{0}+\mathrm{E}[\tilde{z}]-\pi, y_{0}+\mathrm{E}[\tilde{\varepsilon}]\right),
$$

is the maximum amount of money the individual is ready to give up in order to replace the two risks by their expected value. Denoting by $\pi^{1}$ the partial risk premium vis-a-vis the financial risk $\tilde{z}$ only (see eq. (1)) and by $\pi^{2}$ the partial risk premium vis-a-vis the non-financial risk only, we want to compare the total risk premium $\pi$ with the sum of the partial risk premia $\pi^{1}+\pi^{2}$. More precisely we wonder whether the total risk premium is sub-additive ${ }^{6}$. As stated by the proposition below, the concept of cross-risk vulnerability is quite useful to compare the cost of the total risk with the sum of costs of each individual risk.

For simplicity of the exposition and to get rid of the problems of level effects, we assume that both $\tilde{z}$ and $\tilde{\varepsilon}$ are actuarially neutral risks, i.e. $\mathrm{E}[\tilde{z}]=\mathrm{E}[\tilde{\varepsilon}]=0$. We can then state that

Proposition 4. 1. Given $U(x, y)$ is DARA with respect to $x$, the cross-risk vulnerability is a necessary condition for $\pi \leq \pi^{1}+\pi^{2}$,

2. Given $U(x, y)$ is IARA with respect to $x$, the cross-risk vulnerability is a sufficient condition for $\pi \leq \pi^{1}+\pi^{2}$

3. Given $U(x, y)$ is CARA with respect to $x$, the cross-risk vulnerability is a necessary and sufficient condition for $\pi \leq \pi^{1}+\pi^{2}$.

To prove this result, let us first consider $\pi^{1 / 2}$ the conditional risk premium for removing the financial risk $\tilde{z}$ after the agent has already paid to get rid of the non-pecuniary risk $\tilde{\varepsilon}$. It is such that:

$$
\mathrm{E}\left[U\left(x_{0}+\tilde{z}-\pi^{2}, y_{0}\right)\right]=U\left(x_{0}-\pi^{2}-\pi^{1 / 2}, y_{0}\right)
$$

Equations (23) and (24) imply $\pi=\pi^{1 / 2}+\pi^{2}$, so that $\pi \leq \pi^{1}+\pi^{2}$ if and only if $\pi^{1 / 2} \leq \pi^{1}$. Then, if we make explicit the dependence of the $\pi^{1}$ and $\pi^{1 / 2}$ with respect to the initial endowment, we have

$$
\pi^{1}=\pi_{0}^{V}\left(x_{0}, y_{0}\right) \quad \text { and } \quad \pi^{1 / 2}=\pi_{0}^{U}\left(x_{0}-\pi^{2}\left(x_{0}, y_{0}\right), y_{0}\right)
$$

where $\pi_{0}^{V}\left(x_{0}, y_{0}\right)$ is such that $\mathrm{E}\left[V\left(x_{0}+\tilde{z}, y_{0}\right)\right]=V\left(x_{0}-\pi_{0}^{V}\left(x_{0}, y_{0}\right), y_{0}\right)$, with $V$ the derived utility function (3), and $\pi_{0}^{U}\left(x_{0}, y_{0}\right)$ is such that $\mathrm{E}\left[U\left(x_{0}+\tilde{z}, y_{0}\right)\right]=U\left(x_{0}-\pi_{0}^{U}\left(x_{0}, y_{0}\right), y_{0}\right)$.

\footnotetext{
${ }^{6}$ A closely related question is examined by Eeckhoudt and Gollier (2001) for financial risks.
} 
So, the condition $\pi \leq \pi^{1}+\pi^{2}$ is equivalent to $\pi_{0}^{U}\left(x_{0}-\pi^{2}\left(x_{0}, y_{0}\right)\right) \leq \pi_{0}^{V}\left(x_{0}, y_{0}\right)$. Then, given $U$ is CARA in $x, \pi_{0}^{U}\left(x_{0}-\pi^{2}\left(x_{0}, y_{0}\right)\right)=\pi_{0}^{U}\left(x_{0}\right)$ which leads to

$$
\pi \leq \pi^{1}+\pi^{2} \Longleftrightarrow \pi_{0}^{U}\left(x_{0}, y_{0}\right) \leq \pi_{0}^{V}\left(x_{0}, y_{0}\right), \forall x_{0}, y_{0},
$$

which proves the third point of proposition 4 .

Now, given $U$ is IARA in $x, \pi_{0}^{U}\left(x_{0}-\pi^{2}\left(x_{0}, y_{0}\right)\right) \leq \pi_{0}^{U}\left(x_{0}, y_{0}\right)$ since $\pi^{2}\left(x_{0}, y_{0}\right) \geq 0$ provided that $U_{22} \leq 0$. Then, by cross-risk vulnerability, $\pi_{0}^{U}\left(x_{0}, y_{0}\right) \leq \pi_{0}^{V}\left(x_{0}, y_{0}\right)$, so that $\pi_{0}^{U}\left(x_{0}-\pi^{2}\left(x_{0}, y_{0}\right)\right) \leq$ $\pi_{0}^{V}\left(x_{0}, y_{0}\right)$ which proves the second point of proposition 4.

Finally, given $U$ is DARA in $x, \pi_{0}^{U}\left(x_{0}-\pi^{2}\left(x_{0}, y_{0}\right)\right) \geq \pi_{0}^{U}\left(x_{0}, y_{0}\right)$ (still provided that $\left.U_{22} \leq 0\right)$. Then, if $\pi_{0}^{U}\left(x_{0}-\pi^{2}\left(x_{0}, y_{0}\right)\right) \leq \pi_{0}^{V}\left(x_{0}, y_{0}\right)$, we necessarily have $\pi_{0}^{U}\left(x_{0}, y_{0}\right) \leq \pi_{0}^{V}\left(x_{0}, y_{0}\right)$ which proves the first point of proposition 4 .

\section{Conclusion}

In this paper, we have focused on the impact of an unfair non financial background risk on the riskaversion toward another independent financial risk. More precisely, we have defined the notion of crossrisk vulnerability which extends to the case where the background risk is a non financial risk the concept of risk vulnerability introduced by Gollier and Pratt (1996) for additive risks and by Franke et al. (2006) for multiplicative risks. We have then characterized the class of bivariate von Neumann-Morgenstern utility functions that exhibits the above property. In particular, we have shown that the presence of an independent background risk increases the risk aversion towards other independent financial risk, resulting, for example, in an increased demand for insurance.

This work provides several avenues for future researches. First of all, following Franke et al. (2005) we can notice that agents usually have to bear some background risk, but that the level of this risk may change. It would then be worth wondering how an increase in this background risk makes the agent more risk averse. This question, already addressed by Eeckhoudt et al. (1996) and Kimball (1993) for additive background risks, remains open for multi-attributed utility functions.

Second, we would stress that the property of cross-risk vulnerability turns out to be very useful to provide answers to comparative statics exercises for the demand of insurance. This problem will be exposed elsewhere. Besides, in the case of one-dimensional utility functions, risk vulnerability is related 
to the concept of proper risk aversion introduced by Pratt and Zeckhauser (1987), and to the concept of standard risk aversion introduced by Kimball (1993): standardness implies properness and properness implies risk vulnerability. It will be interesting to see how the concepts of proper risk aversion and standard risk aversion can be extended to the case of non financial background risk, and to analyze if they are related to the cross-risk vulnerability.

\section{A Proof of proposition 1.}

Before we prove proposition 1, let us state the following lemma:

Lemma 1. For any function $F$ such that $F(0)=0$ and $F^{\prime}(0)$ exists, the following statements are equivalent

(i) For all $\tilde{\varepsilon}$ such that $\mathrm{E}[\tilde{\varepsilon}]=0, \mathrm{E}[F(\tilde{\varepsilon})] \geq 0$.

(ii) $F(\varepsilon) \geq F^{\prime}(0) \cdot \varepsilon, \quad \forall \varepsilon$.

Proof.

- $(i) \Longrightarrow(i i)$ : Statement $(i)$ holds for any random variable such that $\mathrm{E}[\tilde{\varepsilon}]=0$. In particular, it holds for any $\tilde{\varepsilon}$ such that

$$
\tilde{\varepsilon}=\left\{\begin{array}{l}
\varepsilon_{1}>0, \quad \text { with probability } p, \\
\varepsilon_{2}<0, \quad \text { with probability } 1-p,
\end{array}\right.
$$

where $p$ satisfies $p \cdot \varepsilon_{1}+(1-p) \cdot \varepsilon_{2}=0$. So, by $(i)$, we have $p \cdot F\left(\varepsilon_{1}\right)+(1-p) \cdot F\left(\varepsilon_{2}\right) \geq 0$, or equivalently, dividing by $p \cdot \varepsilon_{1}=-(1-p) \cdot \varepsilon_{2}>0$ :

$$
\frac{1}{\varepsilon_{1}} F\left(\varepsilon_{1}\right) \geq \frac{1}{\varepsilon_{2}} \cdot F\left(\varepsilon_{2}\right), \quad \forall \varepsilon_{1}>0 \text { and } \varepsilon_{2}<0 .
$$

Therefore, letting $\varepsilon_{2} \rightarrow 0$, we have

$$
\forall \varepsilon_{1}>0, \quad \frac{1}{\varepsilon_{1}} F\left(\varepsilon_{1}\right) \geq F^{\prime}(0),
$$

and letting $\varepsilon_{1} \rightarrow 0$, it yields

$$
\forall \varepsilon_{2}<0, \quad \frac{1}{\varepsilon_{2}} F\left(\varepsilon_{2}\right) \leq F^{\prime}(0) .
$$


Since $F(0)$ is obviously larger than or equal to 0 , we obtain:

$$
\forall \varepsilon, \quad F(\varepsilon) \geq F^{\prime}(0) \cdot \varepsilon
$$

- $(i i) \Longrightarrow(i)$ : If $\forall \varepsilon, F(\varepsilon) \geq F^{\prime}(0) \cdot \varepsilon$, then whatever the distribution of $\tilde{\varepsilon}$, we have

$$
\mathrm{E}[F(\tilde{\varepsilon})] \geq F^{\prime}(0) \cdot \mathrm{E}[\tilde{\varepsilon}],
$$

so that

$$
\mathrm{E}[\tilde{\varepsilon}]=0 \Longrightarrow \mathrm{E}[F(\tilde{\varepsilon})] \geq 0 .
$$

Now starting from definition 2, we just have to focus on actuarially neutral background risks. Then, first of all, we notice that expanding condition (9) we get

$$
-A(x, y)\left[U_{1}\left(x, y^{\prime}\right)-U_{12}(x, y) \cdot\left(y^{\prime}-y\right)\right] \geq\left[U_{11}\left(x, y^{\prime}\right)-U_{112}(x, y) \cdot\left(y^{\prime}-y\right)\right], \quad \forall x, y, y^{\prime},
$$

which is equivalent to

$$
f(x, y, \varepsilon) \geq\left.\partial_{\varepsilon} f(x, y, \varepsilon)\right|_{\varepsilon=0} \cdot \varepsilon, \quad \forall x, y \text { and } \varepsilon,
$$

where

$$
f(x, y, \varepsilon)=U_{11}(x, y) \cdot U_{1}(x, y+\varepsilon)-U_{11}(x, y+\varepsilon) \cdot U_{1}(x, y)
$$

with $\varepsilon=y^{\prime}-y$.

Now, considering a cross-risk vulnerable utility function, we have

$$
\frac{U_{11}(x, y)}{U_{1}(x, y)} \geq \frac{\mathrm{E}\left[U_{11}(x, y+\tilde{\varepsilon})\right]}{\mathrm{E}\left[U_{1}(x, y+\tilde{\varepsilon})\right]}
$$

for all $x, y$ and all random variable $\tilde{\varepsilon}$ with zero mean.

Since $U_{1}>0$, the equation above is equivalent to

$$
U_{11}(x, y) \cdot \mathrm{E}\left[U_{1}(x, y+\tilde{\varepsilon})\right]-\mathrm{E}\left[U_{11}(x, y+\tilde{\varepsilon})\right] \cdot U_{1}(x, y) \geq 0 .
$$


Thus, by definition of $f$, we obtain that $U$ is cross-risk vulnerable toward an actuarially neutral background risk if and only if:

$$
\mathrm{E}[f(x, y, \tilde{\varepsilon})] \geq 0,
$$

for all $x, y$ and all random variable $\tilde{\varepsilon}$ with zero mean.

Since, $f(x, y, 0)=0$ for all $x$ and $y$, lemma 1 allows us to assert that the previous relation holds if and only if

$$
f(x, y, \varepsilon) \geq\left.\partial_{\varepsilon} f(x, y, \varepsilon)\right|_{\varepsilon=0} \cdot \varepsilon, \quad \forall x, y \text { and } \varepsilon,
$$

which, by (35) concludes the proof.

\section{References}

Bleichrodt, H., D. Crainich and L. Eeckhoudt. 2003. Comorbidities and the willingness to pay for health improvements. Journal of Public Economics 87 2399-2406.

Bleichrodt, H. and L. Eeckhoudt. 2006. Survival risks, intertemporal consumption, and insurance: The case of distorted probabilities. Insurance: Mathematics and Economics 38, 335-346.

Chalfant, J.A. and I. Finkelshtain. 1993. Portfolio choices in the presence of other risk. Management Science 39 925-936.

Chiu, W.H. 2005. Degree of downside risk aversion and self-protection, Insurance: Mathematics and Economics 36, 93-101.

Courbage, C. 2001. On bivariate risk premia, Theory and Decision 50 29-34.

Doherty, N. and H. Schlesinger. 1983a. Optimal insurance in incomplete markets. Journal of Political Economy 91 1045-1054.

Doherty, N. and H. Schlesinger. 1983b. The optimal deductible for an insurance policy when initial wealth is random. Journal of Business 56 555-565.

Doherty, N. and H. Schlesinger. 1986. A note on risk premiums with random initial wealth. Insurance: Mathematics and Economics 5 183-185.

Eeckhoudt, L. and M. Kimball. 1992. Background risk, Prudence, and the demand for insurance. Contributions to Insurance Economics, Kluwer Academic Publishers, 239-254. 
Eeckhoudt, L. and C. Gollier. 2001. Which shape for the cost curve of risk? The Journal of Risk and Insurance 68 387-402.

Eeckhoudt, L., C. Gollier, H. Schlesinger. 1996. Changes in background risk taking behavior. Econometrica 64, 683-689.

Eeckhoudt, L., C. Gollier, H. Schlesinger. 2005. Economic and Financial Decisions under Risk. Princeton University Press.

Eeckhoudt, L., B. Rey, H. Schlesinger. 2007. A good sign for multivariate risk taking. Management Science 53, 117-124.

Eeckhoudt, L. and H. Schlesinger. 2006. Putting Risk in its Proper Place. American Economic Review 96, 280-289.

Eichner, T. 2008. Mean Variance Vulnerability. Management Science 54, 586-593.

Eichner, T. and A. Wagener. 2003. Variance vulnerability, Background Risks, and Mean-Variance Preferences. The Geneva Papers on Risk and Insurance Theory 28, 173-184.

Epstein, L.G. and S.M. Tanny. 1980. Increasing generalized correlation: A definition and some economic consequences. Canadian Journal of Economics 13 16-34.

Evans, W.N. and W.K. Viscusi. 1991. Estimation of state-dependent utility functions using survey data. Review of Economics and Statistics 73 94-104.

Franke, G., H. Schlesinger and R. C. Stapleton. 2006. Multiplicative Background Risk. Management Science 52 146-153.

Franke, G., R. C. Stapleton and M. G Subrahmanyam. 2005. Incremental Risk Vulnerability. Working Paper.

Gollier, G. 2001. The Economics of Risk and Time. MIT Press, Cambridge, MA.

Gollier, C. and J.W. Pratt. 1996. Risk vulnerability and the tempering effect of background risk. Econometrica 64 1109-1124.

Hotelling, H. 1936. Relations between two sets of variates. Biometrika 28 321-377.

Kihlstrom, R., D. Romer and S. William. 1981. Risk aversion with random initial wealth. Econometrica 49 911-920. 
Kimball, M. S. 1993. Standard risk aversion. Econometrica 61 589-611.

Kischka, P. 1988. Aspects of optimal insurance demand when there are uninsurable risks. Insurance: Mathematics and Economics 7 9-14.

Menegatti, M. 2001. On the conditions for precautionary savings. Journal of Economic Theory 98 189193.

Nachman, D.C. 1982. Preservation of 'more risk averse' under expectations. Journal of Economic Theory 28 361-368.

Pratt, J.W. 1964. Risk aversion in the small and the large. Econometrica 32 122-136.

Pratt, J.W. and R.J. Zeckhauser. 1987. Proper risk aversion. Econometrica 55 143-154.

Quiggin, J. 2003. Background risk in generalized expected utility theory. Economic Theory 22 607-11.

Quiggin, J. and R. G. Chambers. 1998. Risk Premiums and Benefit Measures for Generalized expected utility theories. Journal of Risk and Uncertainty 17 121-137.

Rey, B. 2003. Total and partial bivariate risk premia. Theory and Decision 55 59-69.

Schlesinger, H. 1981. The optimal level of deductibility in insurance contracts. Journal of Risk and Insurance 48 465-481.

Richard, S. 1975. Multivariate risk aversion, utility independence and separable utility functions. Management Science 22 12-21.

Safra, Z. and U. Segal. 1998. Constant Risk Aversion. Journal of Economic Theory 83 19-42.

Sloan, F.A., W.K. Viscusi, H.W. Chesson, C.J. Conover, K. Whetten-Goldstein. 1998. Alternatives approaches to valuing intangible health losses: the evidence of multiple sclerosis. Journal of Health Economics 17 475-482. 